\title{
VLBI ACTIVITIES IN JAPAN AND A PROJECTED SPACE-VLBI PROGRAM
}

\author{
H. Hirabayashi \\ Nobeyama Radio Observatory \\ Tokyo Astronomical Observatory \\ Nobeyama, Minamimaki, \\ Nagano, 384-13, Japan
}

\begin{abstract}
There are three institutes in Japan that are equipped with VLBI facilities. Of these, Nobeyama Radio Observatory started VLBI experiments with astrophysical and astrometric interests. The Institute of Space and Astronautical Science, the Nobeyama Radio Observatory, and the Radio Research Laboratory collaborated with JPL and other institutions for this first space-VLBI test experiment, generating fringes on ground-TDRSS satellite baselines at $\mathrm{S}$ band. A working group formed by scientists of the above three Japanese institutes proposed to ISAS to launch a space-VLBI satellite. The project, called VSOP, for VLBI Space Observatory Program, is described here.
\end{abstract}

\section{INTRODUCTION}

VLBI was first developed in the field of radio astronomy. It is now a basic tool for astrometry and for geodynamics. In Japan, three institutes have VLBI facilities for different purposes. Also, space-VLBI is becoming a reality with the recent successful demonstration of the first space-VLBI test experiment using the TDRSS (Transfer and Data Relay Satellite System) satellite and ground stations. The Russian RADIOASTRON project (Sagdeev 1984; Kardashev and Slysh 1987) is funded, and the Euro-US QUASAT (Schilizzi et al. 1984; Schilizzi 1987) project has been extensively studied. In Japan, researchers at the Nobeyama Radio Observatory (NRO), the Institute of Space and Astronautical Science (ISAS), and the Radio Research Laboratory (RRL) have been discussing the feasibility of a Japanese space-VLBI project (Hirabayashi 1984; Nishimura et al. 1986). For space-VLBI to become operational, coordination of observing, tracking, and data transfer and processing is needed. This paper reports the Japanese activities in VLBI with special attention to space-VLBI.

\section{VLBI STATIONS IN JAPAN}

In Japan, there are several institutes that are now using VLBI technology or in which related projects are considered. 


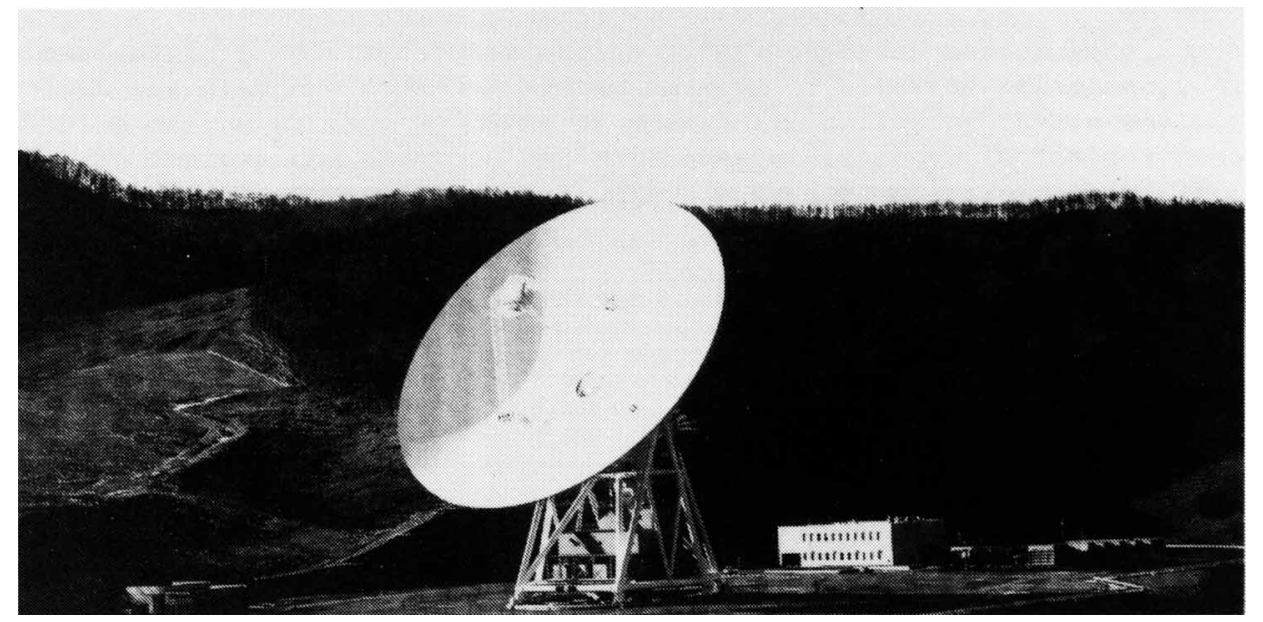

Figure 1. The 64-m antenna of the Usuda Deep Space Center of the Institute of Space and Astronautical Sciences.

RRL operates a 26-m telescope in Kashima. It is equipped with two hydrogen masers, two K3 terminals, and a two-baseline correlator. The K3 terminal and the correlator are Mk III compatible. This equipment was primarily developed by RRL. The RRL group has been actively involved in the Crustal Dynamics Project (CDP) with NASA and is engaged in other projects with many institutes. They operate at $\mathrm{S}$ and $\mathrm{X}$ bands.

The Geographic Survey Institute (GSI) has a 5-m quasi-mobile antenna, primarily stationed at Tsukuba, for obtaining basic data for geographic mapping purposes. $\mathrm{S}$ and $\mathrm{X}$ band frontends, a hydrogen maser, and a $\mathrm{K} 3$ terminal are installed here. The baseline between two points in Japan has already been measured using this station with the collaboration of the RRL group.

$\mathrm{NRO}$ operates a $45-\mathrm{m}$ radiotelescope that is equipped with a hydrogen maser, a Mk III terminal, and two Mk II terminals. The VLBI research activities conducted here will be treated in more detail in the next section.

ISAS has a 61-m deep space station at the Usuda Deep Space Center (UDSC) located in Usuda (Nomura et al. 1985). This antenna, shown in Figure 1, was used for the first space-VLBI demonstration experiment with the TDRSS satellite. For this historical experiment with the Jet Propulsion Laboratory (JPL), NRO and RRL collaborated with ISAS. This experiment will be described in more detail in Section 4. X band VLBI experiments with Kashima and with telescopes abroad have been done by the NRO group here. Mk II terminals, a Mk III terminal, and a rubidium frequency standard were shipped from Nobeyama. ISAS is preparing a K3 terminal, which should be ready by the end of 1987 (T. Nishimura, private communication).

The International Latitude Observatory of Mizusawa (ILOM) proposed project VERA, an acronym for VLBI for Earth Rotation Study and A which is a two-station VLBI observing system for earth rotation study and astrometry (Fujishita 1987). Two antennas of about 35-m and 15-m diameter and related backends are proposed here. ILOM may be combined with the Tokyo Astronomical Observatory within a year. 


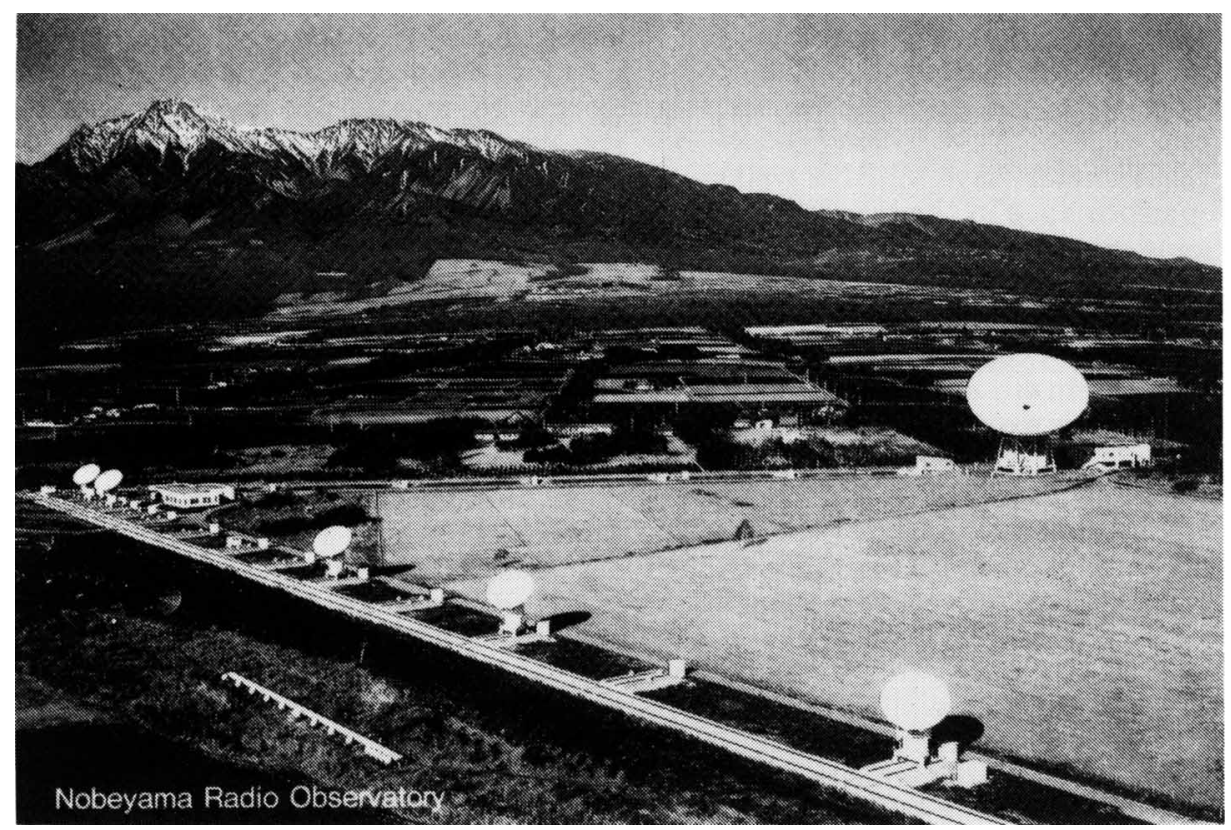

Figure 2. An aerial view of the Nobeyama Radio Observatory.

\section{VLBI AT THE NOBEYAMA RADIO OBSERVATORY}

$\mathrm{NRO}$ is a radio astronomical observatory open to outside users (Akabane et al. 1983). NRO has two major mm-wave radiotelescopes: a 45-m radiotelescope, and a five-element $10-\mathrm{m}$ super-synthesis interferometer. These instruments are shown in Figure 2. For the interferometer, the so-called "FX" architecture was adopted. For this instrument, we developed a 1024-channel spectro-correlator, which has advanced performance compared to conventional cross-correlation type spectrometers (Chikada et al. 1986). This scheme is applicable to a new generation of multi-baseline VLBI spectro-correlators.

For VLBI, the 45-m radiotelescope is used. This telescope is a large-aperture, high-precision millimeter-wave radiotelescope with an rms surface accuracy of about $0.15 \mathrm{~mm}$ and a pointing accuracy of 0.001 degree. A VLG-11 hydrogen maser made by the Smithsonian Astrophysical Observatory, a Mk III recording terminal, and two Mk II terminals are installed. VLBI experiments started in 1983 (Hirabayashi et al. 1985). The VLBI activities include domestic X band experiments, international centimeter and millimeter wave experiments, and spaceVLBI related activities. Domestic VLBI experiments have been done with the Kashima 26-m antenna of RRL for precise coordinate determinations and for VLBI polarimetry. The data tapes were analyzed with the two-baseline correlator at Kashima (Kunimori et al. 1986). The first three baseline experiments were done among the telescopes at Kashima, Nobeyama, and Tsukuba (5-m antenna of GSI) in 1985.

The 22 and $10 \mathrm{GHz}$ bands can be used for VLBI with the 45-m radiotelescope in the microwave region. The 1.4/1.6 and $5 \mathrm{GHz}$ bands are not used any more 
because the primary focus cabin was removed in order to improve the pointing accuracy of the telescope. The $22 \mathrm{GHz}$ water vapor maser line emission was observed with U.S. and European telescopes both in galactic and extragalactic sources.

Millimeter-wave VLBI is well suited to the 45-m radiotelescope because of its high performance in the mm-wave region. Due to the high gain of the telescope and the good uv-coverage afforded by Nobeyama's participation, high quality images can be obtained of the very compact cores of radio galaxies and quasars and of compact line emission regions (Hirabayashi 1981): A $43 \mathrm{GHz}$ experiment with U.S. and European stations generated the first fringes to Nobeyama at millimeter wavelengths in an experiment conducted in May 1986. The Nobeyama-Haystack baseline in this experiment provided a fringe spacing of 0.16 mas, one of the smallest ever obtained. Nobeyama participated in an $89 \mathrm{GHz}$ experiment with four U.S. stations, but no fringes were detected on Nobeyama baselines. The local oscillator chains have been extensively checked and upgraded for mm-wave VLBI.

The first space-VLBI test experiment using the 64-m telescope with the TDRSS satellite proved successful with NRO's full participation with the Usuda Deep Space Center of ISAS (Levy et al. 1986). The NRO group has been interested in space-VLBI, and years of discussion with the scientists in ISAS are continuing. Space-VLBI matters will be discussed in more detail later.

$\mathrm{X}$ band VLBI mapping experiments were done with international telescopes with the $\mathrm{X}$ band front and the VLBI backend of NRO, and the 64-m antenna at Usuda made available by ISAS.

\section{THE TDRSS-OVLBI DEMONSTRATION EXPERIMENT}

Scientists at NRO, ISAS, and RRL are interested in space-VLBI and joint discussions are underway (Hirabayashi 1984; Nishimura et al. 1986). When the idea of the TDRSS-OVLBI (Transfer and Data Relay Satellite System - Orbiting VLBI) demonstration experiment was proposed by JPL, the above scientists were all interested in the experiment. The TDRSS-OVLBI experiment generated close collaboration at the inter-institute level in Japan.

In the early calibration experiments among the ground stations, Mk II terminals were used. Just before that time, the NRO Mk II terminal was completed, and a test experiment for the terminal was done using the 45-m telescope and U.S. telescopes. At the Usuda station, there were no VLBI facilities, and two Mk II terminals, a rubidium clock, a Mk III recording terminal, and other miscellaneous instruments of NRO were shipped to Usuda. Scientists and technicians of NRO installed these instruments on the 64-m antenna and collaborated with the staff of ISAS throughout the experiment.

The Kashima group of RRL participated in the experiment using both the 26-m antenna for reception of the cosmic signals and the K3 correlator for processing the data. They also played an important role in the calibration of the ground stations to phase the telescopes for VLBI use. Also, throughout the TDRSS-OVLBI experiments, the Kashima group observed the sources and recorded tapes.

Fringes were successfully obtained among three stations (TDRSS 4.9-m, Usuda 64-m, and Tidbinbilla 64-m antennas) for three quasars in the JulyAugust 1986 session, but not for the TDRSS-Kashima baseline. The maximum projected baseline obtained was $17,800 \mathrm{~km}$ ( 1.4 times the earth diameter) by the 
TDRSS-Usuda baseline (Levy et al. 1986). Here, the feasibility of space-VLBI was demonstrated and the scheme of transmitting the reference signal from the ground to the space antenna proved iiseful. Figure 3 shows the concept of the TDRSSOVLBI demonstration experiment.

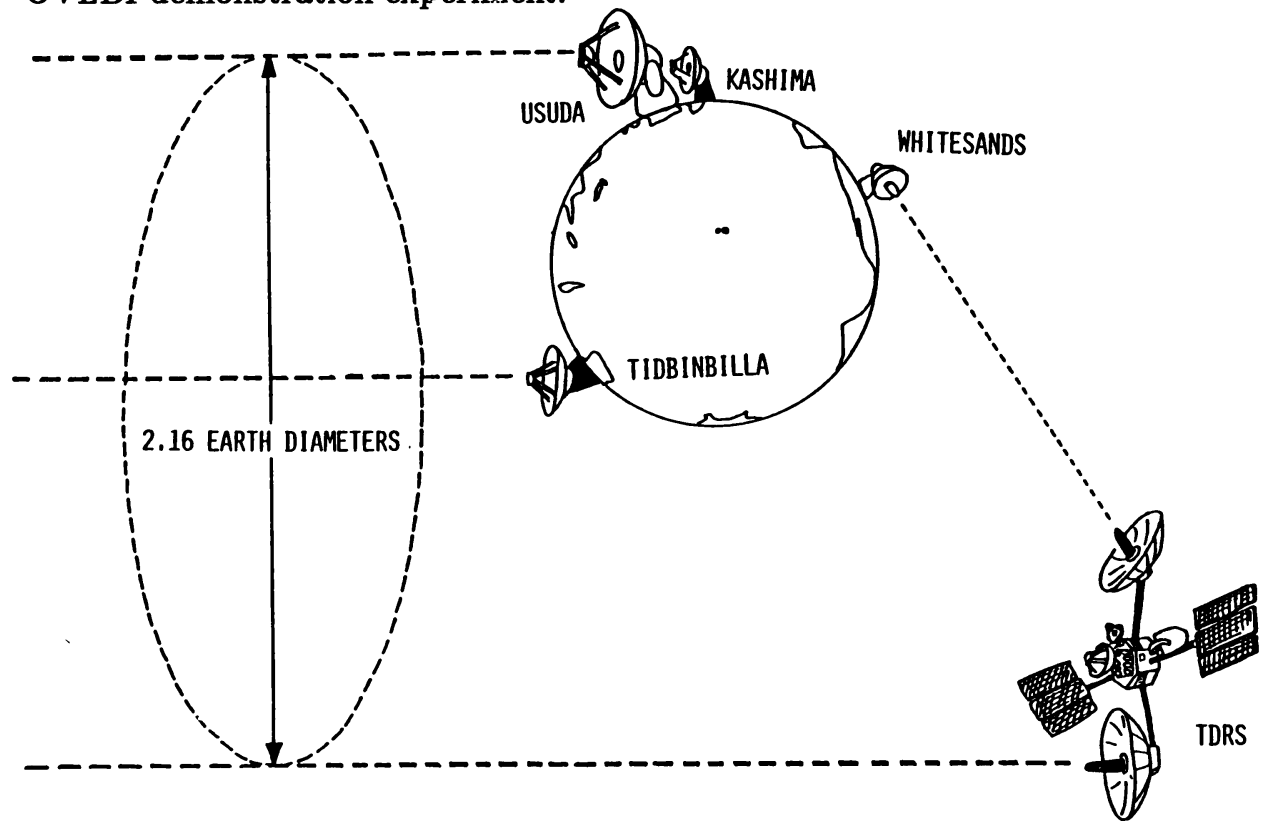

Figure 3. The concept of the TDRSS-OVLBI demonstration experiment.

In the January 1987 session of the experiment, we detected fringes on 23 radio sources out of 25 observed, and in spite of the small gain of the telescopes, the TDRSS $(4.9 \mathrm{~m})$-Kashima $(26 \mathrm{~m})$ pair generated fringes for at least 3C279. The maximum projected baseline obtained was extended to 2.2 times the earth diameter (Linfield et al. 1987). The above experiments were done in $\mathrm{S}$ band. The possibility of observations at $15 \mathrm{GHz}$ is under discussion. Participation of the 45-m radiotelescope at that frequency is possible.

\section{THE VSOP JAPANESE SPACE-VLBI MISSION}

A working group formed in ISAS by scientists from ISAS, NRO, and RRL proposed a Japanese space-VLBI mission, called VSOP, VLBI Space Observatory Program, to ISAS in March 1987 after a two-year feasibility study. The specifications are not yet fixed. Figure 4 shows a rough sketch of the VSOP satellite. For space-VLBI to become operational, cooperation is needed because Japan cannot develop all the items independently of the RADIOASTRON and QUASAT projects.

The best candidate for the observing frequency bands are 5 and $22 \mathrm{GHz}$. The $5-\mathrm{GHz}$ band was selected because it is one of the most active bands for ground-based VLBI. The 22-GHz band was selected because of the importance of water vapor maser line emission, to map the stars of special phase, to obtain the astronomical distance scale, and to measure the rotation of galaxies. 


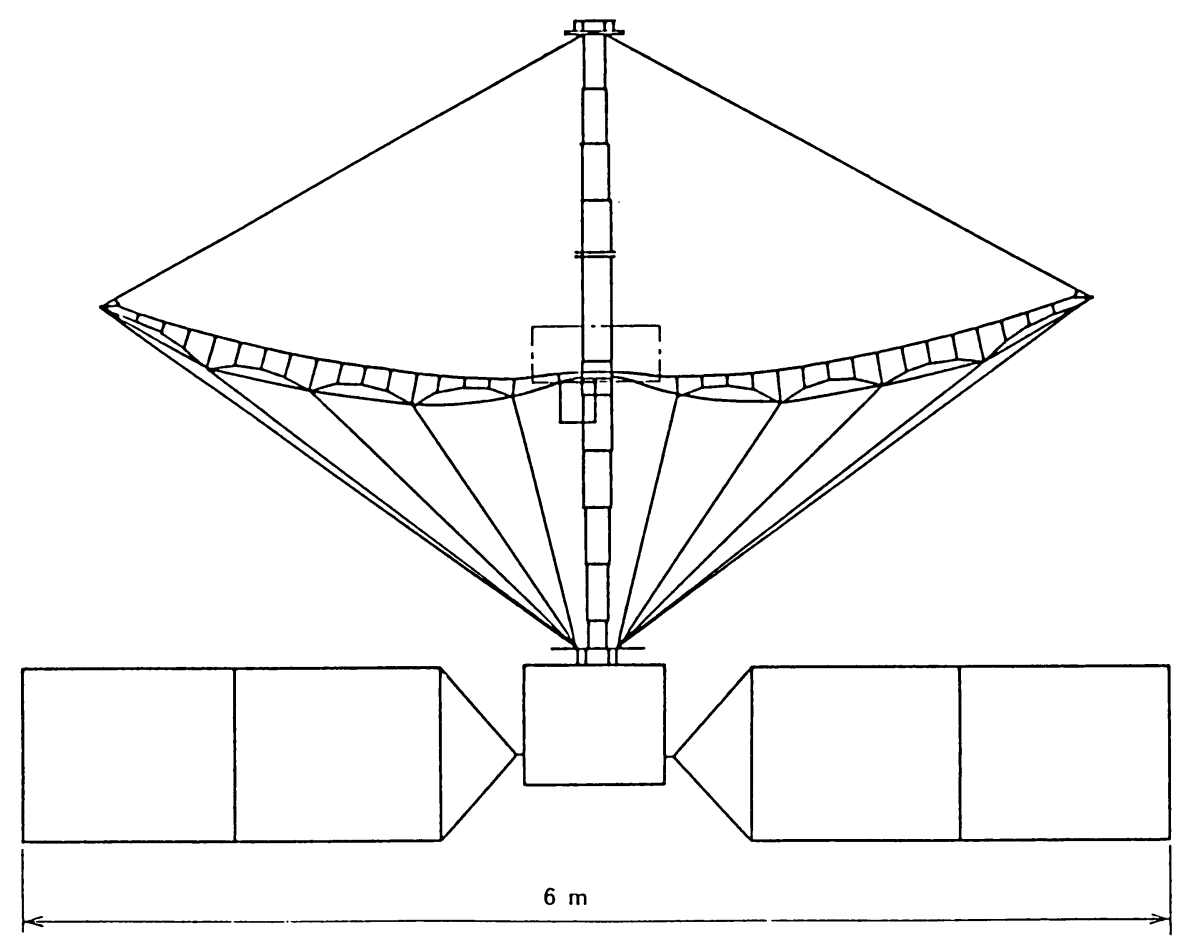

Figure 4. A possible configuration of the VSOP satellite.

We assume that an ISAS rocket is available to launch the VSOP satellite. The M3S-II rocket is the largest existing launching rocket in ISAS and is the same type used for launching the two Japanese Halley probes (Hirao and Itoh 1986). ISAS is considering a more powerful rocket, but it has not been approved yet. Due to the severe limitations of the launching capability, parameters of the payload mass and the satellite orbit were traded off roughly as below. Note that this is a case study where a M3S-II rocket is assumed. The parameters are:

$\begin{array}{lll}\text { inclination } & = & 31^{\circ} \\ \text { perigee altitude } & = & 1000 \mathrm{~km} \\ \text { apogee altitude } & = & 10000 \mathrm{~km} \\ \text { eccentricity } & = & 0.379 \\ \text { satellite mass } & = & 300 \mathrm{~kg}\end{array}$

The M3S-II rocket would be modified to have the extra apogee-kick capability. The payload weight includes all subsystems: the observing and data link, power supply, thermal control, receiving antenna, pointing, satellite structure, and integration system. An effort will be made to make the diameter of the antenna as large as possible, at least larger than $5 \mathrm{~m}$, with the limitation that the mass of the antenna must be less than $100 \mathrm{~kg}$.

The inclination of $31^{\circ}$ is the same as the latitude of the Uchinoura Launching Station of ISAS and was chosen to maximize the payload mass (Tsuboi and 
Hirabayashi 1985). If we fix the orbit as above, the variation of the longitude of the ascending node $(\Omega)$ is $-1.32^{\circ} /$ day, or $-480^{\circ} /$ year. The variation of the argument of the periapsis $(\omega)$ is $2.07^{\circ} /$ day, or $750^{\circ}$ year. In the QUASAT proposal, the former parameter is $-0.22^{\circ} / \mathrm{day}$, and the latter is set to zero by choosing an inclination of $63^{\circ}$. When a more powerful rocket is available in ISAS, then a larger space-VLBI satellite can be considered. The VSOP satellite introduced here is too limited in mass, and a more powerful launching rocket is highly desirable.

A deployable antenna structure is considered. Figure 4 shows one example of the antenna mounted on the satellite, and Figure 5 shows a related example of the satellite in the launching rocket, with the antenna folded up.
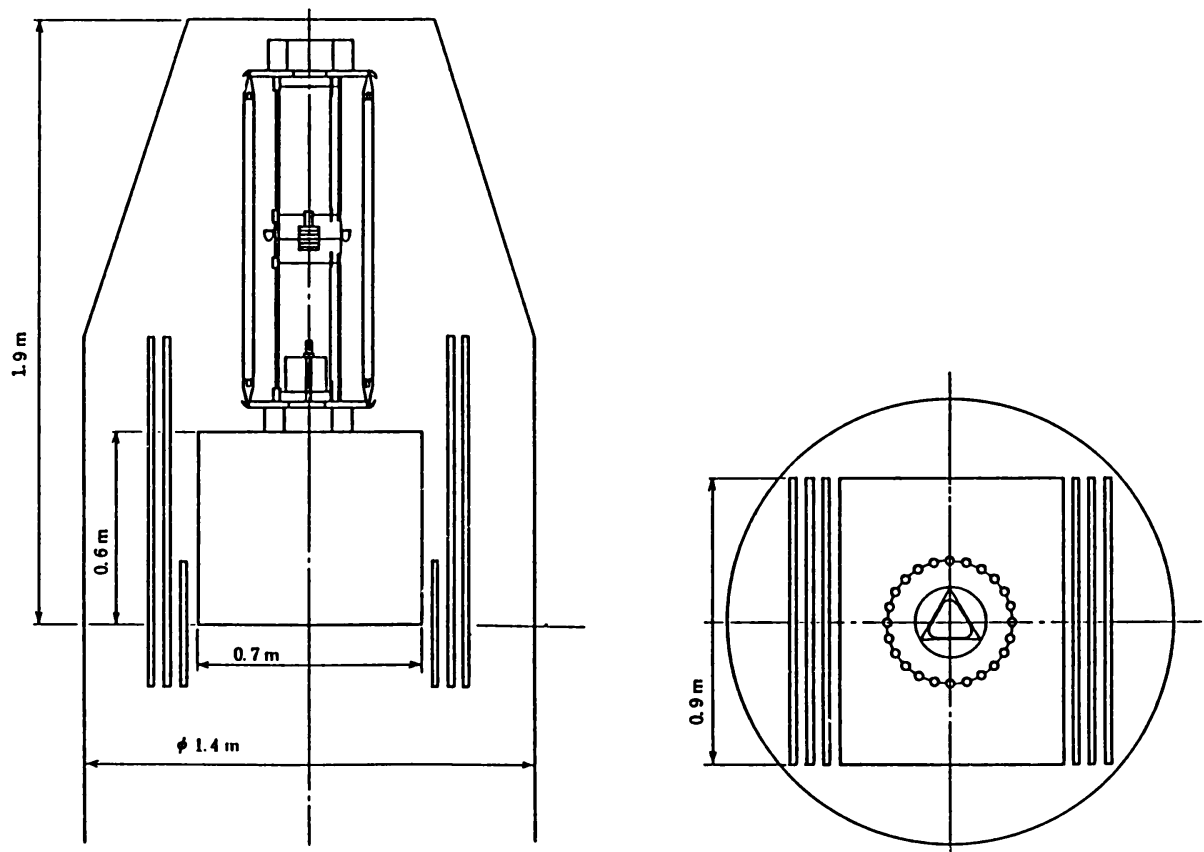

Figure 5. An example of the VSOP satellite folded in the launching rocket.

\section{INTERNATIONAL COLLABORATION AND VSOP}

To make the space-VLBI satellite an effective mission, an array of ground stations for the reception of terrestrial signals from the radio sources as well as other antennas for tracking, for the local signal link, and for the information downlink are needed. Without these ground stations, sensitivity and uv-coverage are limited. These ground stations must be coordinated for the effective utilization of the VLBI satellite. At present, Japan has neither a world-wide ground network for satellite tracking nor a VLBI ground network. Collaboration at the international level is an important item to be discussed. Effective sharing of the orbit and compatibility with other possible space-VLBI missions should also be considered to maximize the efficiency of the satellites. 


\section{CONCLUSION}

VLBI activities and the status of space-VLBI in Japan have been described. Discussion both inside Japan and at the international level is needed to promote an efficient space-VLBI program.

\section{REFERENCES}

Akabane, K., Morimoto, M., Kaifu, N., and Ishiguro, M. 1983, "The Nobeyama Radio Observatory," Sky and Telescope, Dec. 1983, p. 495.

Chikada, Y., et al. 1986, "A Very Fast FFT Spectrum Analyser for Radio Astronomy," in Proceedings of IEEE-IECEJ-ASJ International Conference on Acousties and Signal Processing, April 7-11, 1986, p. 2907.

Fujishita, M., and Hara, T. 1987, "Japanese VLBI Project - VERA," this volume.

Hirabayashi, H. 1981, "International VLBI Array," in Ann. Tokyo Astron. Obs., 2nd Series, 18(2), p. 82.

Hirabayashi, H. 1984, "An Introductory Review for Japanese Space-VLBI Mission," in Proceedings of the Meeting on Space-VLBI, Institute of Space and Astronautical Science, May 26, 1984.

Hirabayashi, H., Inoue, M., Iwashita, H., Morimoto, M., and Tsuboi, M. 1985, "Very Long Baseline Interferometry by $\mathbf{4 5} \mathrm{m}$ Radiotelescope," in Proceedings of International Symposium on Antennas and Propagation, Kyoto, ISAP-85, p. 975.

Hirao, K., and Itoh, T. 1986, "The Planet-A Halley Encounters," Nature, 321, 294.

Kardashev, N. S., and Slysh, V. I. 1987, "RADIOASTRON Project," this volume.

Kunimori, H., et al. 1986, "The Geodetic Results of VLBI Experiment on KashimaNobeyama Baseline," in Proceedings of the Symposium on Applications of Space Technologies to Astronomy and Astrophysics, Tokyo, Jan.-Feb. 1986, p. 138.

Levy, G. S., et al. 1986, "Very Long Baseline Interferometry Observations Using an Orbiting Radio Telescope," Science, 234, 187.

Linfield, R. P., et al. 1987, "Very Long Baseline Interferometry Observations Using the Tracking and Data Relay Satellite as an Orbiting Telescope," this volume.

Nishimura, T., Takano, T., Yamada, T., and Hirabayashi, H. 1986, "Japanese Space VLBI Mission," in Proceedings of the Symposium on Applications of Space Technologies to Astronomy and Astrophysics, Tokyo, Jan.-Feb. 1986, p. 152.

Nomura, T., Hayashi, T., Nishimura, T., Hirosawa, H., and Ichikawa, M. 1985, "Usuda Deep Space Station with $64 \mathrm{~m}$ Diameter Antenna," in Proceedings of 36th IAF Symposium, Stockholm, IAF-85, 381.

Sagdeev, R. Z. 1984, "Some Prospects of Space VLBI," in QUASAT - A VLBI Observatory in Space, Proceedings of a Workshop held at Gross Enzersdorf, Austria (European Space Agency, Noordwijk, The Netherlands), p. 19.

Schilizzi, R. T. 1987, "QUASAT," this volume.

Schilizzi, R. T., Burke, B. F., Jordan, T. F., and Hawkyard, A. "The QUASAT Mission: An Overview," in QUASAT - A VLBI Observatory in Space, Proceedings of a Workshop held at Gross Enzersdorf, Austria (European Space Agency, Noordwijk, The Netherlands), p. 13 (see also p. 27).

Tsuboi, M., and Hirabayashi, H. 1985, "Toward the Optimum Selection of the Orbit of a Japanese Space-VLBI Satellite," Ann. Tokyo Astron. Obs., 2nd Series, 20(3), p. 191. 